\title{
ÂGE À LA MIGRATION DE REPRODUCTION DES GÉNITEURS DE TROIS COHORTES DE GRANDES ALOSES (ALOSA ALOSA) DANS LE BASSIN VERSANT DE LA GARONNE (FRANCE).
}

\author{
P. LAMBERT (1), D. MARTIN VANDEMBULCKE (1), E. ROCHARD (1), \\ J.L. BELLARIVA $(2,3)$, G. CASTELNAUD (1)
}

(1) Cemagref, Unité ressources aquatiques continentales, 50, avenue de Verdun, 33612 CESTAS Cedex, France. E-mail : patrick.lambert@ cemagref.fr

(2) ENSAT, Equipe Environnement aquatique et aquaculture, BP 107 Auzeville, 31326 CASTANET TOLOSAN Cedex, France

(3) adresse actuelle : 8, avenue du roustillou, 31140 MONTBERON, France.

E-mail : bellariva@ libertysurf.fr

Reçu le 27 septembre 2000

Received 27 September, 2000

Accepté le 22 mai 2001

Accepted 22 May, 2001

\section{RÉSUMÉ}

La population de grandes aloses (Alosa alosa) du bassin versant de la Garonne est encore abondante. L'importance et la structure des flux de migration 1994, 1995 et 1996 ont été estimées à partir du suivi des captures de la pêcherie et des passages au niveau d'un obstacle. Des campagnes d'échantillonnage précisent les caractéristiques biologiques des individus. La force des cohortes 1989, 1990 et 1991 est basée sur le suivi des abondances mensuelles des alosons en estuaire.

A partir de ces informations, il a été possible, pour les cohortes 1989 à 1991, de rapporter les effectifs de géniteurs d'un sexe donné, remontant à un âge donné au nombre d'alosons présents en estuaire lors de leur première année.

C'est à 5 ans que remonte le maximum de géniteurs, mâles comme femelles. En moyenne, $5,26 \%$ des alosons en estuaire donnent des femelles de 5 ans et $4,89 \%$ des mâles de 5 ans. Le patron de migration pour les géniteurs de 4 et 6 ans diffère selon le sexe : 2,61 \% des alosons en estuaire deviendront des géniteurs mâles de 4 ans et $0,73 \%$ des mâles de 6 ans. $0,99 \%$ et 2,08 \% des alosons en estuaire donneront respectivement des femelles de 4 et 6 ans. Les entrées de géniteurs de 3 ans et 7 ans restent inférieures à $0,2 \%$ pour les deux sexes. Globalement, $8,40 \%$ des alosons deviennent des géniteurs femelles, $8,33 \%$ des géniteurs mâles.

A noter enfin la forte variabilité de la proportion globale de femelle issue d'une cohorte. $51,0 \%$ des géniteurs de la cohorte 1989 étaient des femelles, 56,8 \% pour la cohorte 1990 et seulement 40,9\% pour la cohorte 1991 .

Mots-clés : Alosa alosa, migration, âge, flux, cohorte. 


\title{
AGE OF MIGRATION FOR SPAWNERS FROM THREE COHORTS OF ALLIS SHAD (ALOSA ALOSA) IN THE GIRONDE WATERSHED (FRANCE).
}

\begin{abstract}
The population of allis shad (Alosa alosa) of the Gironde watershed is still abundant. The abundance and the structure were estimated for the runs 1994, 1995 and 1996. These estimations are based on surveys and samplings (i) of the captures of the fisheries and (ii) of the passages in fish lift. The strength of the cohorts 1989, 1990 and 1991 was evaluated according to the follow-up of monthly abundance of juveniles in estuary.
\end{abstract}

With this information, it was possible, for the cohorts 1989 to 1991 , to calculate the ratio between the number of spawner of one sex, migrating at one age and the number of young of the year (yoy) in estuary.

Maximum of the spawners, males as females, migrate at age 5. On average, $5.26 \%$ of the yoy in estuary will become 5 years old female spawners of and $4.89 \% 5$ years old male spawner. The migration pattern for the adults of 4 and 6 years is different according to the sex. $2.61 \%$ of yoy in estuary will become 4 years old male spawners and $0.73 \% 6$ years old males. $0.99 \%$ and $2.08 \%$ of the juveniles in estuary will respectively give females of 4 and 6 years. The entrance of 3 years and 7 year old spawners remain lower than $0.2 \%$ for the two sexes. Globally, $8.40 \%$ of the yoy in estuary become female spawners, $8.33 \%$ male spawners.

Finally, notice the strong variability of the total proportion of female produced by a cohort. $51.0 \%$ of the spawners from the cohort 1989 were females, $56.8 \%$ from the cohort 1990 and only $40.9 \%$ from the cohort 1991.

Key-words : Alosa alosa, migration, age, run, cohort.

\section{INTRODUCTION}

La population de grandes aloses (Alosa alosa L., 1758) inféodée au bassin versant de la Garonne est considérée comme l'une des plus abondantes sur l'aire de répartition de cette espèce (TAVERNY, 1991). La pêcherie qui lui est associée fait l'objet d'un suivi depuis près de 20 ans (CASTELNAUD et al., 2001) et des comptages sur les frayères et aux premiers obstacles sont réalisés depuis une dizaine d'années (CHANSEAU, comm. pers.). La base de connaissance sur l'écologie de cette population est riche (CASSOULEINS et CASSOU-LEINS, 1981; DAUTREY et LARTIGUE, 1983; TAVERNY, 1991 ; BENGEN, 1992 ; ROCHARD, 1992 ; BELLARIVA, 1998 ; MARTIN VANDEMBULCKE, 1999). Cette population d'alose fait l'objet d'une pêcherie professionnelle dont l'intérêt socio-économique est indéniable. Sur la période 1980-1995, la quantité pêchée annuellement varie de $500 \mathrm{t}$ à $600 \mathrm{t}$ pour une valeur de 6 à 8 millions de francs (CASTELNAUD et ROCHARD, 1997).

Le présent travail a pour objet d'une part de déterminer les structures en âge des géniteurs pour trois saisons de migration en combinant des informations issues de suivis de pêcherie et provenant de suivis des passages au niveau d'un obstacle. II vise, d'autre part, à reconstituer les structures en âge pour trois cohortes. 


\section{MATÉRIEL ET MÉTHODE}

La méthode d'estimation du nombre de juvéniles repose sur l'hypothèse émise par TAVERNY (1991) d'une stabulation en zone estuarienne des alosons dévalant des zones fluviales : la force d'une cohorte correspond au maximum de densité observée en estuaire entre juillet d'une année et juillet de l'année suivante.

LAMBERT et al. (1997) et MARTIN VANDEMBULCKE (1999) en appliquant cette méthode aux données du suivi mensuel de la faune circulante dans l'estuaire de la Gironde (ELIE et ROCHARD, 1994 ; PRONIER et ROCHARD, 1998) trouvent des abondances, de 5,00 millions d'individus pour la cohorte 1989, 3,66 et 4,93 pour celles de 1990 et 1991.

L'estimation de 1991 repose sur quatre transects, répartis entre 30 et $50 \mathrm{~km}$ de la mer, comprenant, chacun, trois stations en surface et trois stations en fond. Pour 1989 et 1990, seul le transect situé à $43 \mathrm{~km}$ de la mer était disponible.

Les échantillonnages ont été effectués à partir d'un navire avançant face au courant de flot pendant $5 \mathrm{mn}$ à une vitesse d'environ 1 nœud. Les échantillons de surface ont été effectués à l'aide de 2 cadres de type "pibalour » portés de part et d'autre du bateau. Leur section est de $4 \mathrm{~m}$ par $1 \mathrm{~m}$. Le maillage étiré est de $18 \mathrm{~mm}$ dans la partie principale de l'engin (ventre et dos) et de 2,8 $\mathrm{mm}$ dans la partie terminale. Les échantillonnages de fond, ont été effectués à l'aide d'un traîneau supra-benthique constitué d'un cadre métallique de $2 \mathrm{~m}$ sur $1,2 \mathrm{~m}$, maintenu à $0,2 \mathrm{~m}$ du fond par des patins. La profondeur varie en fonction des stations entre $3,5 \mathrm{~m}$ et $8,0 \mathrm{~m}$. Le maillage de cet engin est identique à celui des filets utilisés en surface.

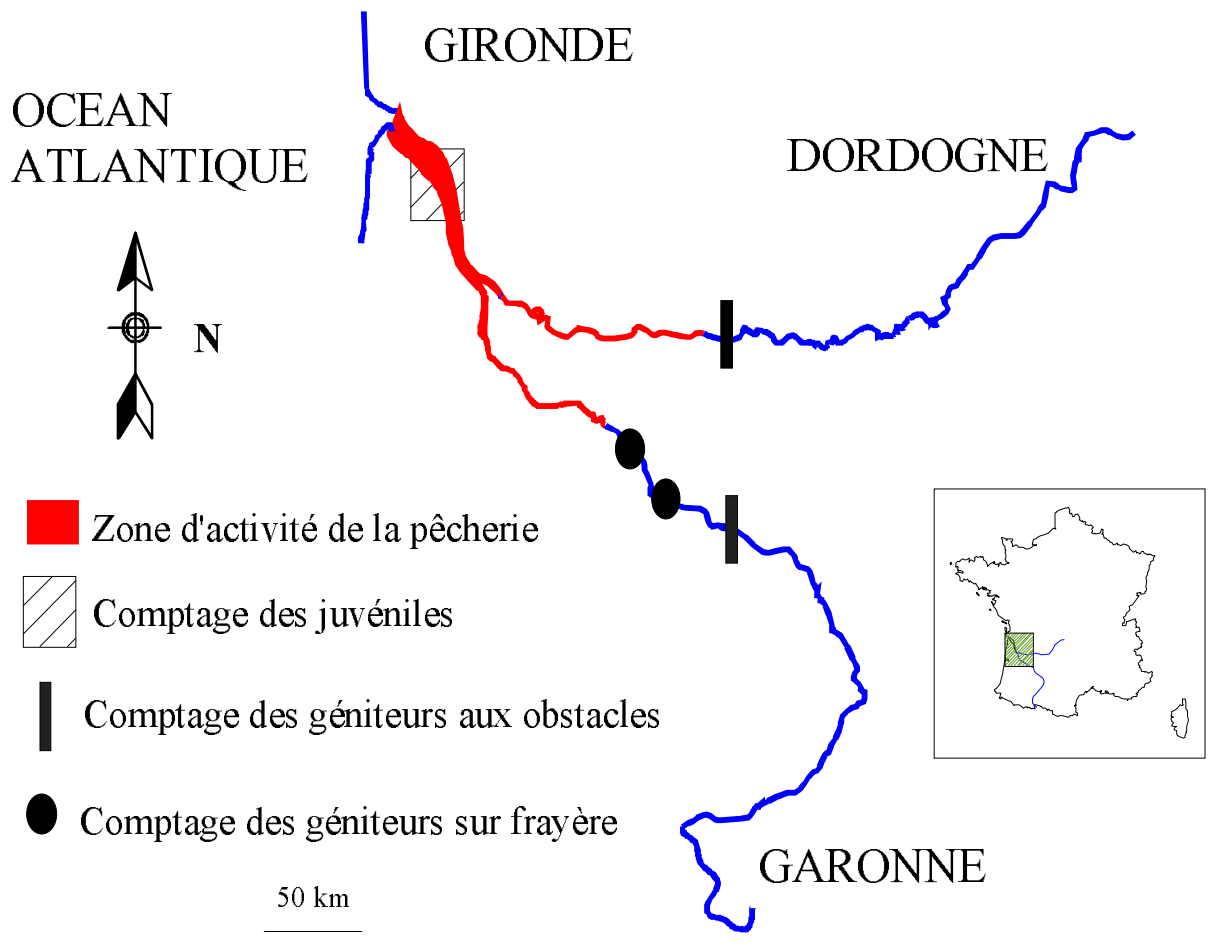

Figure 1

Localisation du système fluvio estuarien Gironde-Garonne-Dordogne.

\section{Figure 1}

Localization of the Gironde-Garonne-Dordogne estuarine system. 
L'estimation du nombre total de géniteurs rentrant en estuaire (MARTIN VANDEMBULCKE, 1999 ; CHANSEAU, comm. pers.) est obtenue par sommation des animaux prélevés par la pêcherie et celles des aloses ayant échappé à la pêche (Tableau I). Le premier élément, désigné par «captures » dans le reste du document, correspond aux estimations des captures totales des pêcheries professionnelle et amateur du bassin de la Garonne (CASTELNAUD et al., 2001). Le second, dénommé « échappement » par la suite, somme des comptages sur les frayères de Garonne en aval du premier obstacle et les comptages aux passes à poissons des premiers obstacles sur les deux fleuves (situé à $110 \mathrm{~km}$ de la confluence sur la Dordogne, à $200 \mathrm{~km}$ sur la Garonne). L'estimation du nombre de géniteurs sur les frayères à l'aval du premier obstacle sur la Dordogne a été extrapolée à partir des résultats obtenus sur la Garonne (CHANSEAU, comm. pers.).

\section{Tableau I}

Estimations des captures et de l'échappement utilisées.

\section{Table I}

Used estimation of captures and escapement.

\begin{tabular}{|c|c|c|c|c|c|c|c|c|}
\hline \multirow[b]{2}{*}{ Année } & \multicolumn{4}{|c|}{ Captures ${ }^{(1)}$} & \multicolumn{3}{|c|}{ Echappement ${ }^{(2)}$} & \multirow{2}{*}{$\begin{array}{c}\text { Taux } \\
\text { d'exploitation }\end{array}$} \\
\hline & Estuaire & Garonne & Dordogne & TOTAL & Garonne & Dordogne & TOTAL & \\
\hline 1994 & 49595 & 170019 & 120610 & 340224 & 158846 & 211621 & 370467 & $47,9 \%$ \\
\hline 1995 & 50034 & 190704 & 86053 & 326791 & 206656 & 264543 & 471198 & $41,0 \%$ \\
\hline 1996 & 41805 & 201691 & 110186 & 353682 & 184706 & 295002 & 479708 & $42,4 \%$ \\
\hline
\end{tabular}

(1) d'après CASTELNAUD et al., 2001 ; (2) d'après CHANSEAU, comm. pers.

La structure en âge et le pourcentage de femelles dans le flux de migrants pendant les saisons 1994-1996 ont été estimés en combinant des informations associées au suivi de la pêcherie professionnelle et des données récoltées au niveau du premier obstacle sur l'un des axes fluviaux, la Garonne. Un plan d'échantillonnage stratifié par compartiment géographique (estuaire, Garonne et Dordogne) (Figure 1) des animaux capturés par la pêcherie a été mis en place (MARTIN VANDEMBULCKE, 1999) pour les trois saisons de migration considérées. Les pêcheurs utilisent des filets tramail dont le maillage étiré de la nappe centrale est de $110 \mathrm{~mm}$. L'échantillonnage de la fraction non capturée a été réalisé, pour 1995 et 1996, dans la goulotte de rejet de l'ascenseur à poissons de la centrale hydroélectrique de Golfech sur la Garonne avec une épuisette de maillage $60 \mathrm{~mm}$ (BELLARIVA, données non publiées).

Le sexe a été déterminé par pression des flancs. Pour l'estimation de l'âge, des écailles ont été prélevées entre la ligne latérale et l'aplomb de la nageoire dorsale (CASSOU-LEINS et CASSOU-LEINS, 1981) puis préparées selon un protocole standardisé (GAZEAU et al., 2001). La méthode d'interprétation des écailles (estimation de l'âge et marques de ponte) est celle classiquement employée par différents auteurs (CASSOU-LEINS et CASSOU-LEINS, 1981 ; MENNESSON-BOISNEAU, 1990 ; TAVERNY, 1991). Deux lectures ont systématiquement été effectuées, complétées par une troisième lorsque les deux premières donnaient des résultats différents. 
Les structures en sexe et en âge des captures (Tableau II) ont été calculées en pondérant les proportions de femelles et de géniteurs des différents âges issues des échantillonnages biologiques par les captures réalisées durant la période encadrant chaque prélèvement biologique. Pour se faire, les captures annuelles par compartiment ont été réparties entre les différentes périodes au prorata des déclarations des seuls pêcheurs nous communiquant leur prise journalière.

En 1994, aucun prélèvement biologique n'était disponible en estuaire. Nous avons estimé les différentes proportions en agrégeant les résultats obtenus en Garonne et en Dordogne cette même année.

\section{Tableau II}

Résultats des prélèvement biologiques sur les captures pour les saisons 1994, 1995 et 1996.

\section{Table II}

Biological sampling results of capture during 1994, 1995 and 1996 season.

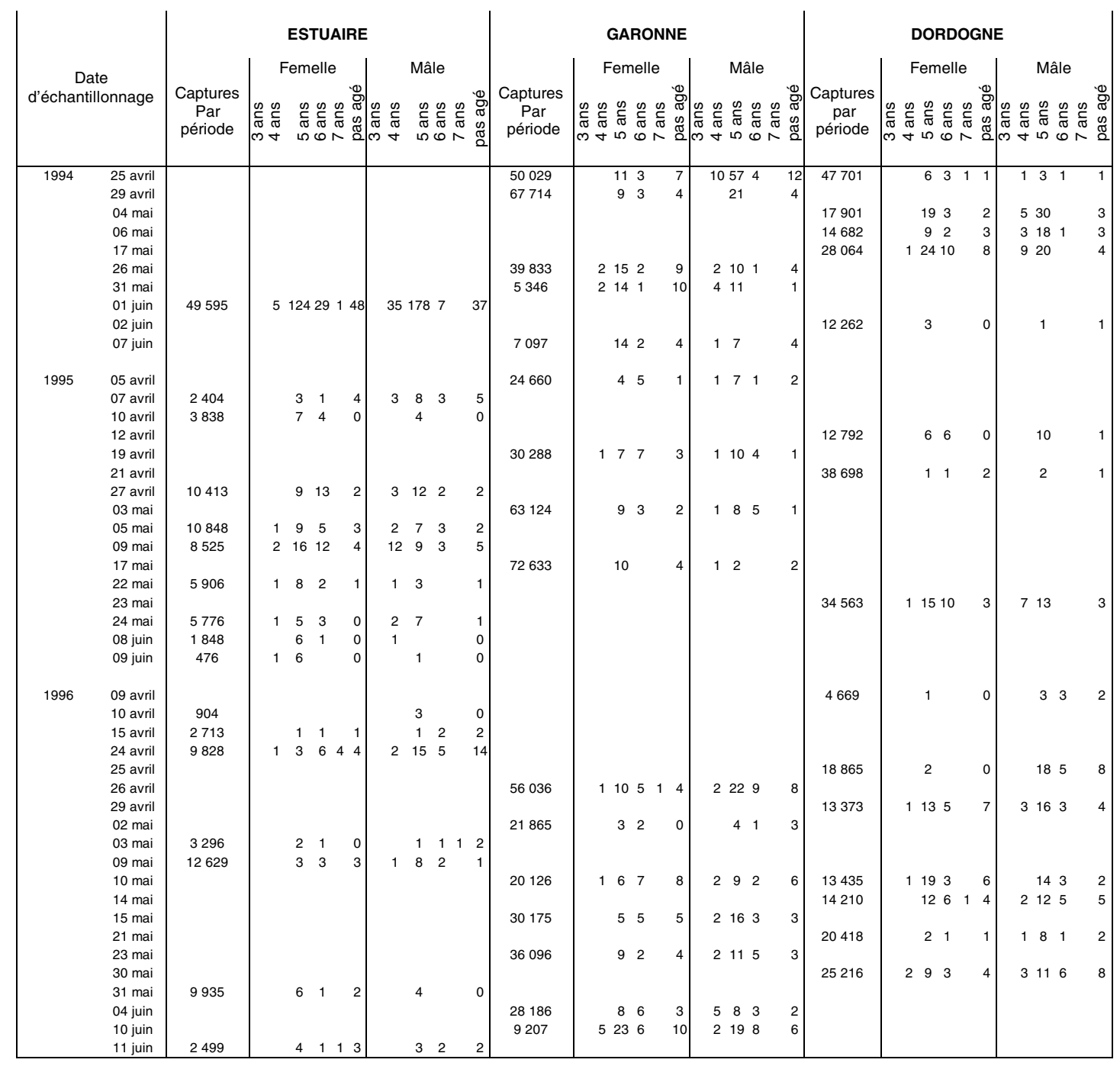


Les structures en sexe et en âge au niveau du premier obstacle sur la Garonne (Tableau III) ont été calculées pour les années 1995 et 1996 par une méthode analogue en considérant les comptages journaliers au niveau de la passe (association MiGaDo, données non publiées). Nous avons fait l'hypothèse que ces proportions étaient valides pour l'ensemble des animaux échappant à la pêcherie (i.e. sur les frayères de Garonne à l'aval du premier obstacle et sur l'axe fluvial Dordogne).

\section{Tableau III}

Résultats des prélèvement biologiques sur l'échappement pour les saisons 1994, 1995 et 1996.

Table III

Biological sampling results of escapement during 1994, 1995 and 1996 season.

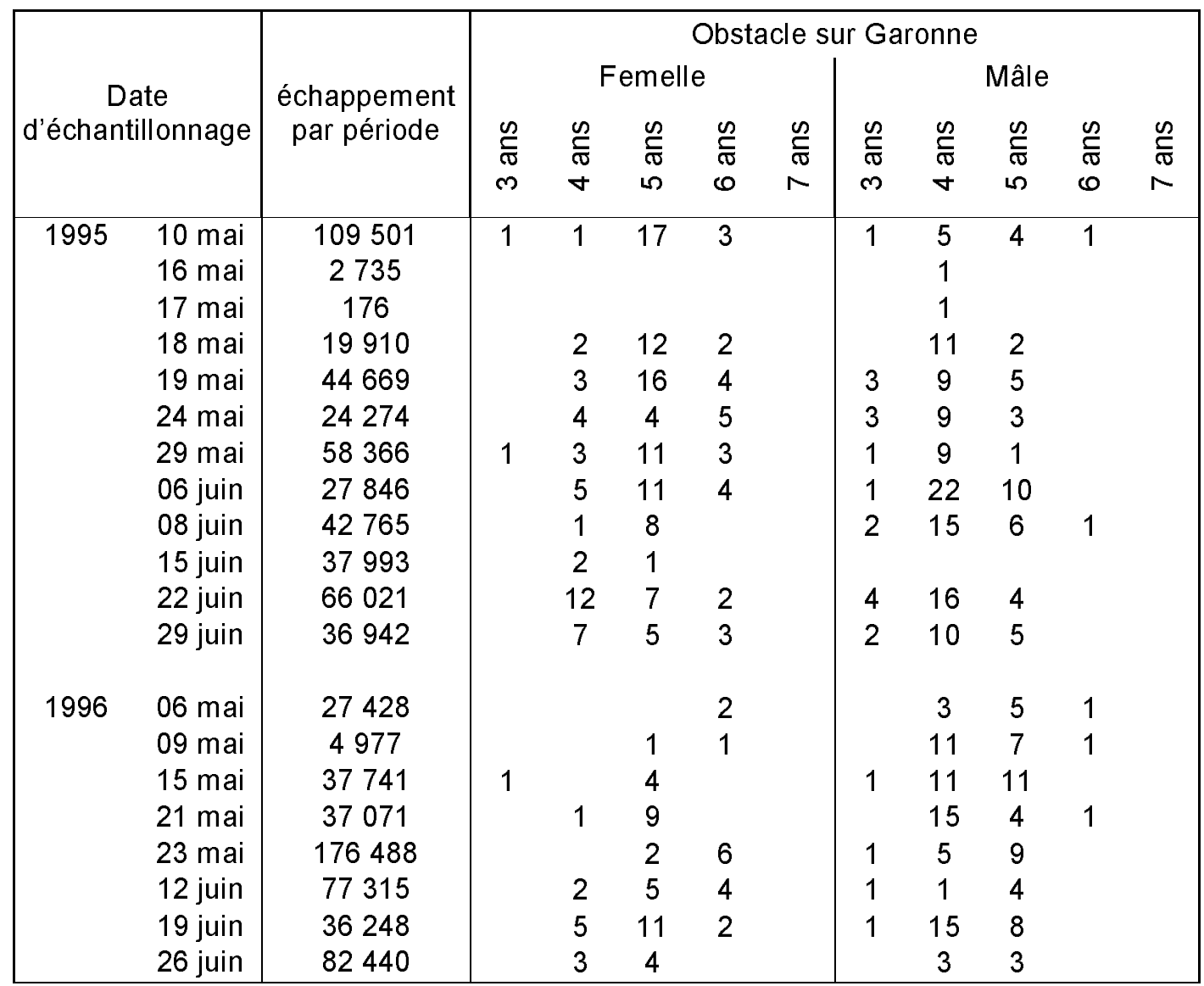

L'extrapolation de la structure démographique du flux échappant à la pêcherie lors de la saison 1994 est basée sur le respect du taux d'exploitation globale en approchant les taux d'exploitation par âge d'un sexe donné. Le taux d'exploitation est défini, pour un type d'individus comme étant le rapport entre le nombre de poissons pêchés sur le total des animaux de ce type migrant une saison. Ce taux pour un sexe et un âge donnés correspond, en fait, à la sélectivité de la pêcherie pour les animaux considérés.

Plus précisément, la méthode de reconstitution se fait en trois étapes :

- à partir des captures par âge et par sexe mesurées en 1994, on calcule des effectifs théoriques de l'échappement pour les femelles et mâles d'âge 4, 5 et 6 ans en appliquant la moyenne des taux d'exploitation aux âges et par sexe trouvé en 1995 et 1996. 
- pour les âge 3 qui échappent systématiquement à la pêcherie, l'effectif de l'échappement est calculé à partir de la moyenne des proportions de ces animaux dans les flux de migrants 1995 et 1996.

- les effectifs théoriques sont ensuite corrigés pour retrouver l'effectif global estimé de l'échappement cette année là.

Enfin, les paramètres démographiques concernant les âges les moins représentés manquants pour la reconstitution des cohortes 1990 mais surtout 1989 et 1991 ont été estimés par la moyenne des valeurs correspondantes mesurées lors des saisons 1994 à 1996.

Le taux d'itéroparité pour les années considérées est faible ( $3 \%$; MARTIN VANDENBULCKE, 1999), contrairement aux observations des années antérieures (17,9\% pour les femelles et $9,1 \%$ pour les mâles ; TAVERNY, 1991). Nous n'avons pas tenu compte de ce phénomène dans les calculs des âges de remontée des cohortes 1989, 1990 et 1991.

Les traitements statistiques ont été réalisés à l'aide du logiciel Systat 8.0. Les comparaisons des structures en sexe et en âge ont été effectuées par des tests du $\chi^{2}$ pondérés par les passages et des captures totales.

\section{RÉSULTATS}

Les effectifs aux âges des flux de migrants lors des saisons 1994, 1995 et 1996 sont synthétisés dans le Tableau IV. Ils sont à la base des interprétations qui suivent.

\section{Tableau IV}

Résumé des structures démographiques des flux de migrant 1994, 1995 et 1996.

\section{Table IV}

Summary of demographic structure of 1994, 1995 and 1996 migration run.

\begin{tabular}{|c|c|c|c|c|c|c|c|c|c|c|}
\hline \multirow{3}{*}{\multicolumn{2}{|c|}{$\begin{array}{c}\text { Type } \\
\text { d'individus }\end{array}$}} & \multicolumn{3}{|c|}{ Saison 1994} & \multicolumn{3}{|c|}{ Saison 1995} & \multicolumn{3}{|c|}{ Saison 1996} \\
\hline & & \multicolumn{2}{|c|}{ Effectif } & \multirow[b]{2}{*}{$\begin{array}{c}\text { Taux } \\
\text { d'exploitation }\end{array}$} & \multicolumn{2}{|c|}{ Effectif } & \multirow[b]{2}{*}{$\begin{array}{c}\text { Taux } \\
\text { d'exploitation }\end{array}$} & \multicolumn{2}{|c|}{ Effectif } & \multirow[b]{2}{*}{$\begin{array}{c}\text { Taux } \\
\text { d'exploitation }\end{array}$} \\
\hline & & 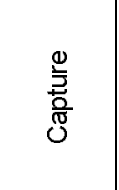 & 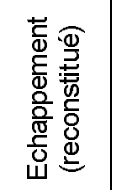 & & 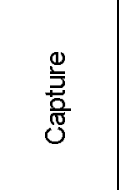 & 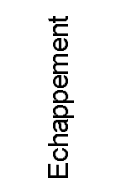 & & 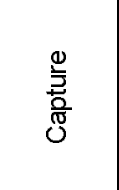 & 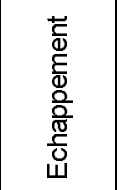 & \\
\hline \multirow{5}{*}{ 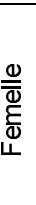 } & 3 ans & 0 & 2051 & 0,00 & 0 & 5331 & 0,00 & 0 & 1348 & 0,00 \\
\hline & 4 ans & 4153 & 34567 & 0,11 & 3266 & 72486 & 0,04 & 4990 & 33672 & 0,13 \\
\hline & 5 ans & 118285 & 100395 & 0,54 & 131703 & 152975 & 0,46 & 88893 & 89696 & 0,50 \\
\hline & 6 ans & 31867 & 25275 & 0,56 & 53843 & 34666 & 0,61 & 44733 & 71182 & 0,39 \\
\hline & 7 ans & 3226 & 0 & 1,00 & 0 & 0 & & 2653 & 0 & 1,00 \\
\hline \multirow{5}{*}{$\frac{\Phi}{\frac{\omega}{\omega 0}}$} & 3 ans & 0 & 11328 & 0,00 & 0 & 22576 & 0,00 & 0 & 14432 & 0,00 \\
\hline & 4 ans & 23171 & 109503 & 0,17 & 21708 & 129036 & 0,14 & 19361 & 118334 & 0,14 \\
\hline & 5 ans & 151446 & 86366 & 0,64 & 96793 & 49513 & 0,66 & 144908 & 147077 & 0,50 \\
\hline & 6 ans & 8076 & 983 & 0,89 & 19478 & 4614 & 0,81 & 47456 & 3966 & 0,92 \\
\hline & 7 ans & 0 & 0 & & 0 & 0 & & 687 & 0 & 1,00 \\
\hline
\end{tabular}


Pour les deux saisons observées, 1995 et 1996, la proportion de femelles est comparable dans l'échappement et dans les captures ( $p$ Yates = 0,345 pour 1995 et $=0.757$ pour 1996) (Figure 2). En 1994, par reconstitution, les femelles étaient légèrement moins nombreuses dans l'échappement que dans les captures. Ces valeurs conduisent, compte tenu des effectifs respectifs des captures et de l'échappement, a une proportion globale dans le flux de migrants de 45,0 \% de femelles en $1994,56,9 \%$ en 1995 et 40,5\% en 1996.

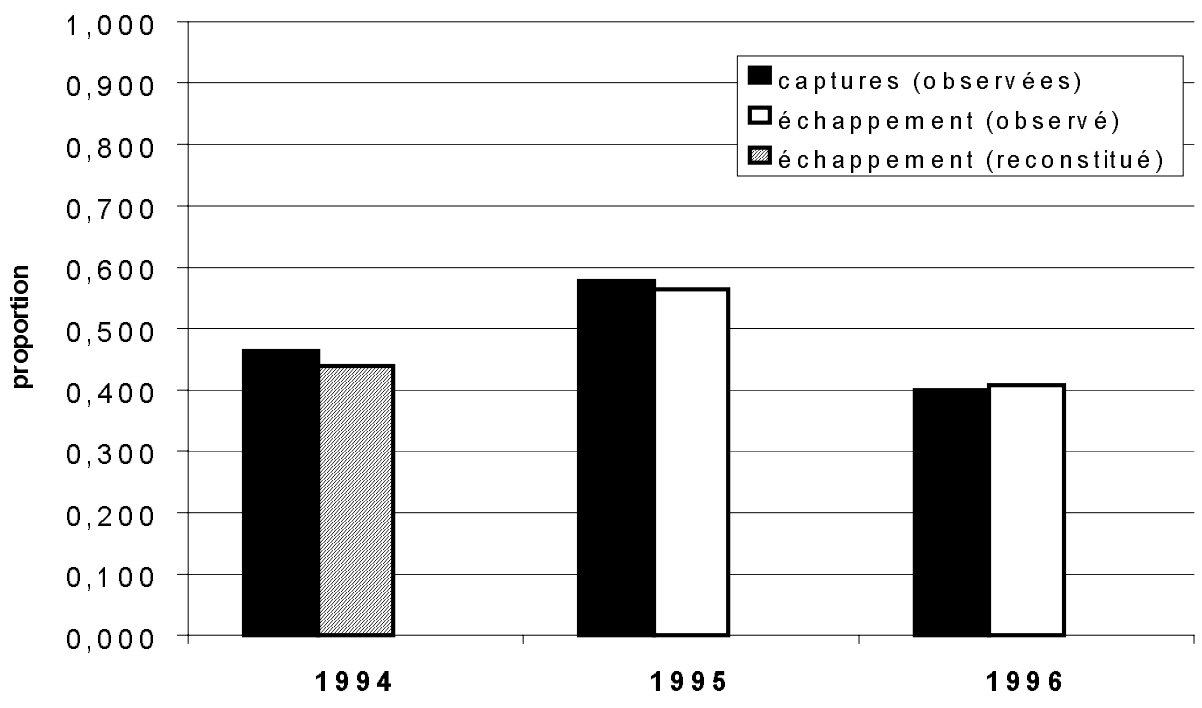

Figure 2

Proportion de femelles dans les flux migrant en 1994, 1995 et 1996.

\section{Figure 2}

Female proportion in the runs 1994, 1995 and 1996.

Le flux de migrant est constitué de femelles principalement de 5 ans et dans une moindre mesure de 6 ans, et de mâles également de 5 ans et dans une moindre mesure de 4 ans (Figure 3). Des différences significatives dans les structures en âge sont enregistrées entre les sources d'information pour les deux sexes et les deux saisons observées ( $p \chi^{2} \leq 0,000$ sauf pour les femelles de 1996 où $p \chi^{2} \leq 0,009$ ). La prise en compte de l'échappement conduit à amoindrir la dissymétrie des âges de migration pour les femelles et à renforcer celle des mâles. Les structures globales des flux de 1995 et 1996 s'avèrent au final être significativement différentes $\left(\mathrm{p} \chi^{2} \leq 0,000\right.$ pour les deux sexes). Le flux 1996 se caractérise par une sur abondance des femelles de 6 ans aux dépends de celles de 5 ans et par une sur abondance des mâles de 5 ans aux dépends de ceux de 4 ans. Pour la saison 1994, le flux reconstitué présente une sur représentation des mâles de 5 ans. 

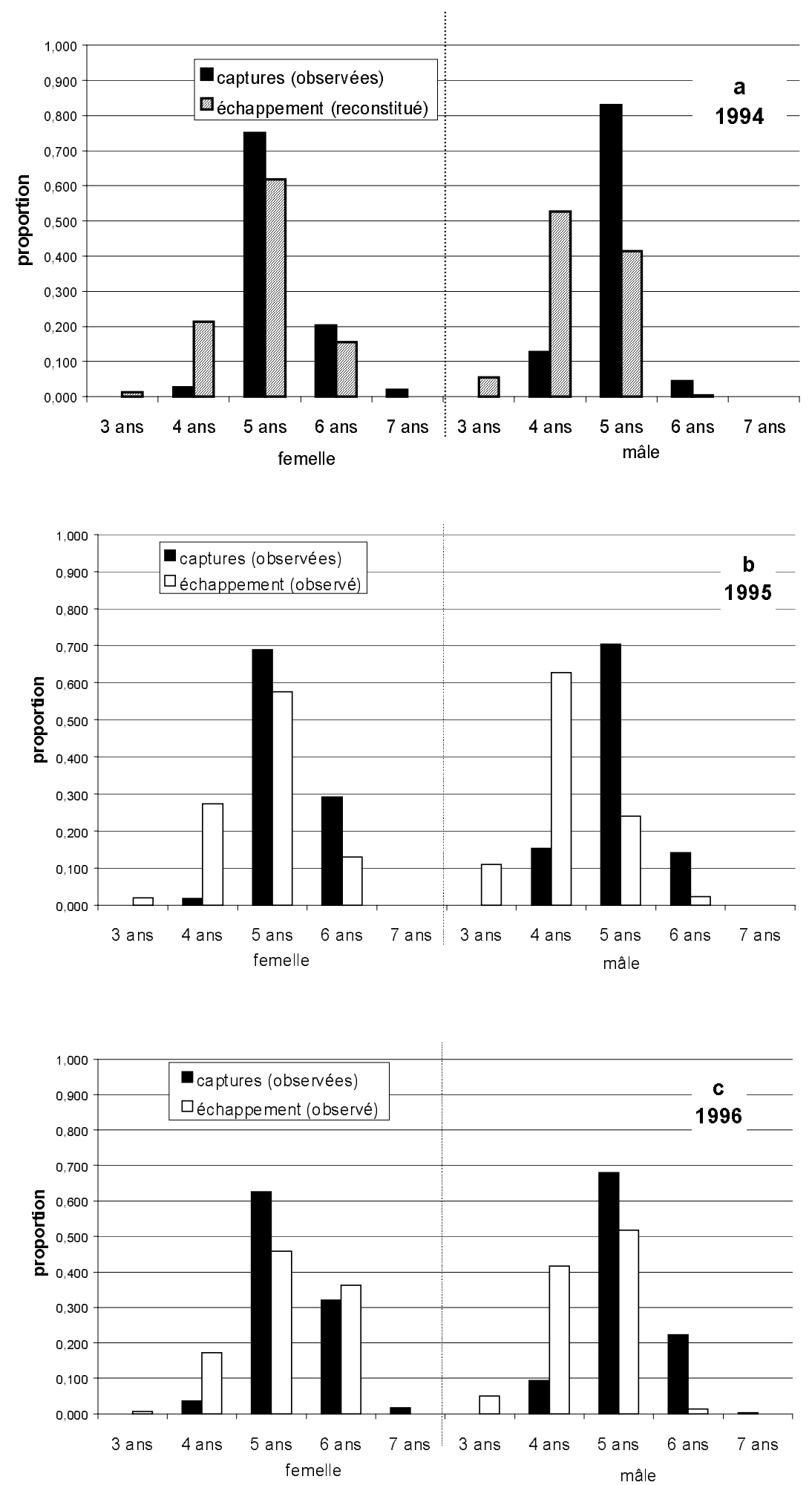

Figure 3

Structure en âge du flux migrant en 1994 (a), en 1995 (b) et en 1996 (c).

Figure 3

Age structure in the runs 1994 (a), 1995 (b) and 1996 (c). 
Pour les saisons 1995 et 1996, la pêcherie a capturé près de $42 \%$ des femelles migrantes. Le taux d'exploitation augmente en fonction de l'âge des animaux, pour les deux sexes ( $\tau$ de Kendall $=0,837$ pour les femelles ; =0,956 pour les mâles) (Figure 4), les mâles de 4 ans étant moins capturés que les femelles du même âge, le contraire étant observé pour les géniteurs de 6 ans.

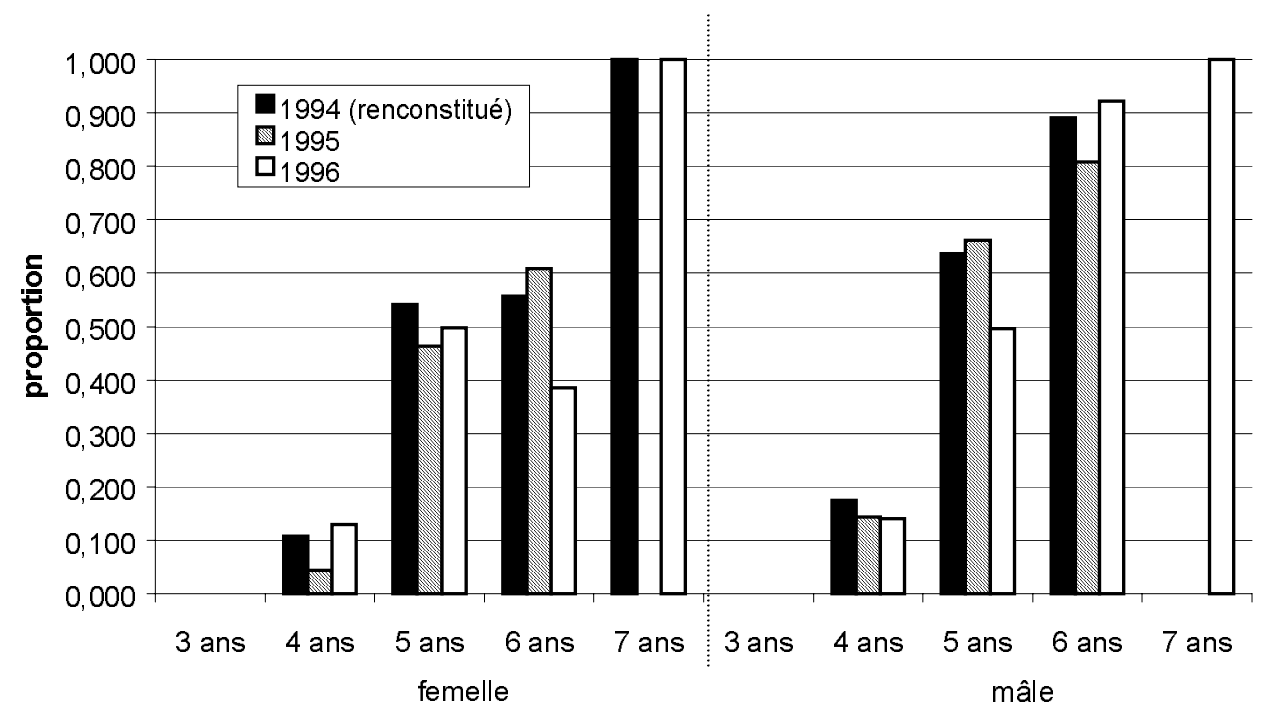

\section{Figure 4}

Taux d'exploitation des flux migrant en 1994, 1995 et 1996.

\section{Figure 4}

\section{Exploitation rate of the runs 1994, 1995 and 1996.}

Pour une cohorte, c'est à 5 ans que remonte le maximum de géniteurs, mâles comme femelles (Figure 5). En moyenne sur les trois cohortes suivies, 5,26 \% des alosons en estuaire donnent des femelles de 5 ans, 4,89 \% des mâles de 5 ans. Le patron moyen de migration pour les géniteurs de 4 et 6 ans est différent pour les mâles et les femelles. $2,61 \%$ des alosons en estuaire deviendront des géniteurs mâles de 4 ans et $0,73 \%$ des mâles de 6 ans. $0,99 \%$ des alosons en estuaire et 2,08 \% donneront respectivement des femelles de 4 et 6 ans. Les entrées de géniteurs de 3 ans et 7 ans restent inférieures à 0,2 $\%$ pour les deux sexes. La sommation de ces différents pourcentages permet d'avancer que $9,44 \%$ des alosons deviendront des géniteurs femelles, 8,50 \% des géniteurs mâles. La cohorte 1990 s'individualise par une forte entrée des femelles de 5 ans, de mâles et femelles de 6 ans et une plus faible entrée de mâles de 5 ans. Un déficit de femelles de 5 ans et une sur représentation des géniteurs mâles de 4 ans caractérisent la cohorte 1991.

A noter enfin que la proportion globale de femelles varie fortement selon les cohortes : $51,0 \%$ des géniteurs de la cohorte 1989 étaient des femelles, 56,8 \% pour la cohorte 1990 et seulement 40,9\% pour la cohorte 1991 . 




Figure 5

Age à la reproduction pour les aloses des cohortes 1989, 1990 et 1991.

Figure 5

Age of reproduction for shads from cohorts 1989, 1990 and 1991.

\section{DISCUSSION}

Contrairement aux travaux précédents (MENNESSON-BOISNEAU, 1990 ; TAVERNY 1991 ; MARTIN VANDEMBULCKE, 1999), nous avons dépassé l'examen de flux pour une analyse effective des cohortes. Cela revient en fait à ne plus supposer que l'image donnée par le suivi d'un seul flux renseigne sur l'évolution d'une cohorte au cours de son histoire (notion de pseudo-cohorte utilisée dans l'analyse séquentielle des populations, (BRETHES et O'BOYLE, 1990)). En particulier, cette approche simplifiera les recherches futures sur les causes de variabilité des âges à la maturité. En effet, l'hypothèse de fluctuations négligeables du recrutement ne sera plus nécessaire pour analyser les résultats. Rappelons que la force des trois cohortes étudiées a varié de 3,6 millions à 5 millions d'alosons en estuaire (MARTIN VANDEMBULCKE, 1999).

Toutefois, dans l'immédiat, le patron de migration issu de notre analyse par cohorte est cohérent avec celui décrit précédemment dans le cas du bassin de la Garonne par des analyses classiques par flux (TAVERNY, 1991 ; MARTIN VANDEMBULCKE, 1999). C'est toujours principalement des géniteurs de 5 ans qui quittent les eaux marines pour participer à la reproduction auxquels viennent s'ajouter des mâles de 4 ans et des femelles de 6 ans. II s'inscrit dans le gradient latitudinal de vieillissement des âges à la maturité proposé par MESSESSON-BOISNEAU et al., 2000. Par contre, nous infirmons la différence de un an dans les âges de remontée des deux sexes, telle qu'annoncée comme règle générale par ces mêmes auteurs.

L'approche par cohorte implique bien évidemment des moyens d'investigation plus importants. Dans notre cas de reconstitution de 3 cohortes, outre les 3 années de suivi biologique et d'estimation des flux de migrants, communs avec l'approche par flux, il a été nécessaire de recourir à l'estimation des flux de migrants et de l'abondance des juvéniles en estuaire pendant 3 années supplémentaires. 
Le fait d'utiliser pour l'établissement des structures en âge, non seulement des informations issues de la pêcherie mais également provenant de l'échappement, permet de prendre en compte dans les calculs des géniteurs de 3 ans qui échappent systématiquement à la pêcherie. II permet également d'augmenter la contribution des individus de 4 ans, femelles comme mâles dans la composition des flux. Corollairement, il conduit à minimiser les proportions de mâles de 5 ans, en particulier pour la cohorte 1990 . Par contre, les structures en sexe ne sont que très peu modifiées par l'intégration des données issues du suivi de l'échappement puisque les sex-ratio dans les captures et dans l'échappement sont voisins, au moins pour les deux saisons observées. Cette prise en compte de deux sources d'informations justifient la nécessité de mettre au point une méthode d'extrapolation de la structure en âge de l'échappement à partir de celles des captures, dans la mesure où une dizaine d'années de suivi biologiques sont disponibles uniquement sur les captures.

Nous avons choisi de pondérer les proportions des différents âges par la somme des captures (ou des comptages aux passes) encadrant le prélèvement biologique plutôt qu'un simple cumul sur l'ensemble de la saison comme dans MARTIN VANDEMBULCKE (1999). Là encore, le patron de migration n'est pas remis en cause mais les différences entre années sont modifiées. En particulier, les proportions de femelles de 6 ans en 1995 sont réévaluées au profit de celles de 5 ans. Cette pondération conduit à infirmer la différence de sex-ratio entre les captures et l'échappement mise en évidence pour le flux 1996 par MARTIN VANDEMBULCKE (1999). En effet, si la proportion des femelles dans les captures a été peu modifiée $(40,1 \%$ au lieu de $40,3 \%)$, leur part dans l'échappement a été largement augmentée (40,8\% au lieu de 34,1\%).

Nous avons fait le postulat que les structures en sexe et en âge des animaux échantillonnés au niveau de la passe sur la Garonne étaient valides pour l'ensemble des aloses échappant à la pêcherie, à savoir les individus frayant à l'aval de cet ouvrage sur cet axe ainsi que tous ceux qui atteignent les frayères sur la Dordogne. La justification de ce postulat est surtout d'ordre utilitariste: nous avons souhaité utiliser au maximum les données disponibles. Nous pouvons tout de même avancer que les structures en sexe et en âge ne sont pas significativement différentes entre les captures de Garonne et celle de Dordogne (MARTIN VANDEMBULCKE, 1999). Mais cette impossibilité à détecter une différence pourrait provenir d'une sélectivité des engins de pêche. En tout cas, l'extrapolation des données pour la Garonne à celles pour la Dordogne suppose l'acceptation du postulat d'une unique et même population sur les deux axes fluviaux ou éventuellement, dans la cas de deux populations, d'un parfait synchronisme des histoires de vie de leurs cohortes successives. Par ailleurs, l'extrapolation des structures en âge d'un secteur donné à l'ensemble d'un linéaire serait mise en défaut s'il s'avérait que la répartition des géniteurs le long d'un axe migratoire était influencée par une variabilité individuelle des capacités migratoires, fonction de la longueur et du stade de maturité (TRUMP et LEGGETT, 1980 ; JONSSON et al., 1991, LEONARD et MCCORMIC, 1999) et donc probablement fonction de l'âge.

Le taux d'exploitation global de la pêcherie est de 47,9 \% en 1994, 41,0 \% en 1995 et $41.4 \%$ en 1996. L'originalité de saison 1994 ne s'explique pas par une variation de l'effort de pêche puisqu'une stabilité des efforts est observée pour les trois années considérées (10 965 jours de pêche en 1994, 11009 jours en 1995 et 10387 jours en 1996) (CASTELNAUD et al., 2001).

Les pourcentages de femelles capturées par la pêcherie en 1995 et 1996 sont très voisins (41,6 \% et 41,9\%). II en est de même pour les mâles. Les pourcentages $(40,1 \%$ en 1995 , et $42,8 \%$ en 1996) sont d'ailleurs du même ordre de grandeur que ceux des femelles. Même si les taux d'exploitation aux âges des mâles sont supérieurs à ceux des femelles au même âge, la structure en âge des deux sexes peut conduire, comme en 
1995, à un taux d'exploitation des mâles, tous âges confondus, plus faible que celui des femelles.

L'interprétation en termes de sélectivité des engins des taux d'exploitation reste problématique. Les différences observées entre les mâles et les femelles contredit la sélectivité dépendant du sexe mise en évidence par MARTIN VANDEMBULCKE (1999), il s'agit maintenant des mâles qui ont un taux d'exploitation plus élevé, conséquence indirecte de l'augmentation de la proportion de femelles dans l'échappement. Pourtant, une pêche plus efficace des mâles est peu vraisemblable puisque la capturabilité des filets augmente a priori avec la circonférence des individus (HAMLEY et RÉGIER, 1973; HAMLEY, 1975 ; HENDERSON et WONG, 1991) qui est certainement plus faible pour ce sexe compte tenu du plus faible volume des gonades mâles que femelles. De même, l'augmentation avec l'âge du taux d'exploitation pourrait simplement s'expliquer par une augmentation de la taille et donc une rétention plus grande par les filets. Cette hypothèse est réfutée par l'analyse de MARTIN VANDEMBULCKE (1999) qui montre qu'en Garonne, les aloses de 5 et 6 ans sont significativement plus grandes dans l'échappement que dans les captures et donc que la pêcherie ne capturerait pas nécessairement les animaux les plus grands.

Les taux d'exploitation aux âges pour les sexes sont comparables entre 1995 et 1996. Il était donc justifié de recourir à une valeur moyenne de ces taux d'exploitation pour reconstituer la structure en âge de l'échappement pour les animaux de 4 à 7 ans de la saison 1994. En l'absence d'exploitation des aloses de 3 ans, nous avons estimé le nombre d'aloses de 3 ans en prenant la moyenne des proportions de ces animaux dans les flux 1995 et 1996. Toutefois, ces hypothèses, liées à la variabilité des taux considérés d'une saison à l'autre, conduisent à une surestimation de près de $25 \%$ de l'échappement global pour la saison 1994, comparé à celui observé sur les passes et les frayères. Ceci nous a conduit à répartir cette erreur sur les différentes classes d'âge en réduisant au prorata des effectifs.

Ce travail montre que le nombre, la variété et la fiabilité des données utilisées, peuvent avoir des conséquences importante sur le résultat final. II apparaît donc indispensable d'avoir recours à une démarche qualité (GAZEAU et al., 2001, CASTELNAUD et al., 2001) pour limiter les erreurs liées à l'échantillonnage et laisser s'exprimer essentiellement la variabilité du phénomène naturel. Enfin, il est important de rappeler que ce travail repose sur la méthode d'estimation des alosons basée sur une hypothèse de sédentarisation des alosons en estuaire qui mériterait d'être éprouvée.

\section{CONCLUSIONS}

Nous avons défini une méthode de reconstitution de la structure globale du flux à partir de données biologiques les plus fréquentes sur ce bassin. Même si elle n'est pas pleinement satisfaisante, elle permet de déduire la structure de l'échappement à partir des données issues de la pêcherie.

Nous avons pu décrire le patron de migration de trois cohortes. Globalement, $8,40 \%$ des alosons deviennent des géniteurs femelles, $8,33 \%$ des géniteurs mâles. C'est à 5 ans que remonte le maximum de géniteurs : $5,26 \%$ des alosons en estuaire donnent des femelles et $4,89 \%$ des mâles de cet âge. Par contre, la proportion de géniteurs de 4 et 6 ans est différente selon le sexe avec une sur représentation des males de 4 ans (2,61 \% des alosons en estuaire deviendront des géniteurs mâles de 4 ans contre $0,99 \%$ des femelles du même âge) et une sur-représentation des femelles de 6 ans $(2,08 \%$ contre $0,73 \%$ ). Les entrées de géniteurs de 3 ans et 7 ans restent inférieures à $0,2 \%$ pour les deux sexes. 
Nous sommes potentiellement en mesure de reconstruire la chronologie de la migration d'une dizaine de cohortes puisque le suivi biologique entrepris en 1994 sur les captures a été poursuivi jusqu'en 2000. II est donc envisageable de rechercher des causes de variation de ces histoires de vie, en particulier de voir si certaines années se sont traduites par une mortalité importante de toutes les cohortes présentes en mer, ou si d'autres sont caractérisées par un retard de migration compensé l'année suivante, si l'âge de maturité dépend de la densité en mer comme chez l'alose feinte (APRAHAMIAN et LESTER, 2001).

Les valeurs moyennes des proportions aux âges des entrées en estuaire sont les paramètres d'un outil de prévision des retours de géniteurs en estuaire à partir des estimations des alosons dévalant. Cet outil permettra d'anticiper les fluctuations de l'abondance du stock annuel et donnera la possibilité de prendre les mesures de gestion adéquates. II pourra alors être utile dans le cadre d'une gestion responsable de cette ressource, au même titre qu'une base de connaissances sur la biologie, l'écologie et la dynamique de population de la grande alose, et que des procédures de suivi et de diagnostic de l'état de la population (MARTIN VANDEMBULCKE, 1999).

\section{BIBLIOGRAPHIE}

APPRAHAMIAN M.W., LESTER S.M., 2001. Variation in the age at first spawning of female twaite shad (Alosa fallax fallax) from the River Severn, England. Bull. Fr. Pêche Piscic., 362/363, 941-951.

BELLARIVA J.L., 1998. Contribution à l'étude du déroulement de la migration et de la reproduction de la grande alose (Alosa alosa L.) en Garonne : étude prospective de la dévalaison des juvéniles. Thèse de doctorat, Ecole Nationale Polytechnique de Toulouse, $113 \mathrm{p}$.

BENGEN G.S.H., 1992. Suivi de la maturation gonadique des aloses, Alosa alosa L., lors de leur migration en Garonne. Thèse de doctorat, Institut National Polytechnique de Toulouse, $86 \mathrm{p}$.

BRETHES J.C., O'BOYLE R.N., 1990. Méthodes d'évaluation des stocks halieutiques. Centre international d'exploitation des océans, Halifax (Nouvelle-Ecosse, Canada), $963 \mathrm{p}$.

CASSOU-LEINS F., CASSOU-LEINS J.J., 1981. Recherches sur la biologie et l'halieutique des migrateurs de la Garonne et principalement de l'alose : Alosa alosa L. Thèse de doctorat, Institut National Polytechnique de Toulouse, $382 \mathrm{p}$.

CASTELNAUD G., ROCHARD E., 1997. Surveillance halieutique de l'estuaire de la Gironde : suivi statistique 1995 - étude de la faune circulante 1996. Rapport Cemagref de Bordeaux / EDF, 152 p.

CASTELNAUD G., ROCHARD E., LE GAT Y., 2001. Analyse de la tendance de l'abondance de l'alose (Alosa alosa) en Gironde à partir de l'estimation d'indicateurs halieutiques sur la période 1977-1998. Bull. Fr. Pêche Piscic., 362/363, 989-1015.

DAUTREY R., LARTIGUE J.P., 1983. Recherche sur la migration des aloses (Alosa alosa) et des truites de mer (Salmo trutta) en Garonne (site de Golfech). Thèse de doctorat, Institut National polytechnique de Toulouse, $212 \mathrm{p}$.

ELIE P., ROCHARD E., 1994. Migration des civelles d'anguilles (Anguilla anguilla L.) dans les estuaires, modalité du phénomène et caractéristiques des individus. Bull. Fr. Pêche Piscic, 335, 81-98.

GAZEAU C., LEFRANÇOIS T., ROCHARD E., 2001. Mise en place d'une démarche qualité dans le cadre de l'échantillonnage d'une population de grande alose (Alosa alosa). Bull. Fr. Pêche Piscic, 362/363, 1017-1024.

HAMLEY J.M., 1975. Review of gillnet selectivity. J. Fish. Res. Board Can., 32 (11), 19431969. 
HAMLEY J.M., REGIER H.A., 1973. Direct estimates of gillnet selectivity to walleye (Stizostedion vitreum vitreum). J. Fish. Res. Board Can., 30, 817-830.

HENDERSON B.A., WONG J.L., 1991. A method for estimating gillnet selectivity of walleye (Stizostedion vitreum vitreum) in multimesh multifilament gill nets in lake Erie, and its application. Can. J. Fish. Aquat. Sci., 48, 2420-2428.

JONSSON N., HANSEN L.P., JONSSON B., 1991. Variation in age, size and repeat spawning of adult Atlantic salmon in relation to river discharge. J. Anim. Ecol., 60, 937-947.

LAMBERT P., PRONIER O., ROCHARD E., MARTIN-VANDEMBULCKE D., 1997. Analyse des données issues du suivi de la faune circulante aux abords de la centrale de Braud-et-Saint-Louis - Cas des juvéniles d'aloses (Alosa alosa), des crevettes blanches (Palaemon longirostris) et des civelles d'anguilles (Anguilla anguilla). Rapport d'étude Cemagref-EDF n²8, $36 \mathrm{p}$.

LEONARD J.B.K., MCCORMICK S.D., 1999. Effects of migration distance on whole-body and tissue-specific use in American shad (Alosa sapidissima). Can. J. Fish. Aquat. Sci., 56, 1159-1171.

MARTIN VANDEMBULCKE D., 1999. Dynamique de population de la grande alose (Alosa alosa L., 1758) dans le bassin versant Gironde-Garonne-Dordogne (France): analyse et prévision par modélisation. Thèse de doctorat, Ecole Nationale Polytechnique de Toulouse, $115 \mathrm{p}$.

MENNESSON-BOISNEAU C., 1990. Recherches sur les aloses (Alosa sp.) dans le bassin de la Loire. Thèse de doctorat, Université de Rennes I, $105 \mathrm{p}$.

MENNESSON-BOISNEAU C, APRAHAMIAN M.W., SABATIÉ M.R., CASSOU-LEINS J.J., 2000. Remontée migratoire des adultes. In : BAGLINIÈRE J.L., ELIE P. (Eds.) Les aloses (Alosa alosa et Alosa fallax spp.) Cemagref Editions /Inra Editions, 55-72.

PRONIER O.,ROCHARD E., 1998. Fonctionnement d'une population d'éperlan (Osmerus eperlanus, osmériformes osmeridae) située en limite méridionale de son aire de répartition, influence de la température. Bull. Fr. Pêche Piscic, 350-351, 479-497.

ROCHARD E., 1992. Mise au point d'une méthode de suivi de l'abondance des amphihalins dans le système fluvio-estuarien de la Gironde, application à l'étude écobiologique de l'esturgeon Acipenser sturio. Université de Rennes I, 296 p.

TAVERNY C., 1991. Contribution à la connaissance de la dynamique des populations d'aloses (Alosa alosa et Alosa fallax) dans le système fluvio-estuarien de la Gironde: pêche, biologie, écologie. Etude particulière de la dévalaison et de l'impact des activités humaines. Thèse de doctorat, Université de Bordeaux I, $451 \mathrm{p}$.

TRUMP C.L., LEGGETT W.C., 1980. Optimum swimming speeds in fish : the problem of currents. Can. J. Fish. Aquat. Sci., 37, 1086-1092. 
